\title{
The Neutrophilic Leukocyte in Wound Repair
}

\author{
A STUDY WITH ANTINEUTROPHIL SERUM
}

\author{
David M. Simpson and Russell Ross \\ From the Department of Pathology, School of Medicine, University of \\ Washington, Seattle, Washington 98195
}

A B S T R A C T The role of the neutrophilic leukocyte in wound healing was investigated by observing the progress of repair in the absence of these cells. Circulating neutrophils were eliminated in guinea pigs by the administration of antineutrophil serum (ANS) $24 \mathrm{hr}$ before wounding and by daily injections throughout a 10 day period of healing. Control animals received normal rabbit serum at the same dose levels and times. The wounds consisted of six linear incisions in the dorsal skin of the animals.

The contents of 24-hr neutropenic and control wounds were compared by quantitating the major cellular and extracellular wound components using a histometric technique. At $24 \mathrm{hr}$, there were no differences between control and neutropenic wounds in the per cent of total wound volume occupied by mononuclear leukocytes and fibrin. The neutropenic wounds had no neutrophils, had a significantly decreased volume of fluid space, and an increased volume of red cells, as compared with controls. The differences in numbers of erythrocytes and amount of fluid space in these two sets of wounds may be related to substances within neutrophils that promote lysis of erythrocytes or affect vascular permeability.

In spite of the lack of neutrophils in the ANS-treated animals during the 10 days of healing, no differences were observed between the control and neutropenic wounds relative to the rate of wound debridement or the extent of repair. The wounds from the two groups of animals were identical in cellularity and degree of connective tissue formation.

These observations support the notion that neither wound debridement nor the formation of granulation tissue are dependent upon the presence of neutrophils. A neutrophil response in early wounds is not an essential antecedent to the infiltration of monocytes, as suggested by previous investigations.

Received for publication 7 January 1972 and in revised form 28 February 1972.

\section{INTRODUCTION}

The inflammatory response resulting from tissue injury or wounding is characterized by a rapid accumulation of numerous polymorphonuclear neutrophilic leukocytes $(\mathrm{PMN})^{1}$ in aseptic wounds within the first $1-2$ days $(1,2)$. By the 3rd day, the number of neutrophils begins to decrease, and mononuclear phagocytes, immature fibroblasts, and capillaries begin to appear, together with newly formed collagen fibrils $(1,3)$.

For many years it has been thought that the inflammatory process, in addition to its protective function, may somehow be involved in the initiation or stimulation of fibrosis $(4,5)$. In 1921, Carrel $(6,7)$ suggested that inflammatory cells, probably neutrophils, contain growthpromoting substances termed "trephones," which stimulate fibroblasts in the connective tissue surrounding wounds. He observed that irritated wounds healed faster than wounds protected by a dressing and attributed this difference to the presence of greater numbers of inflammatory cells in the irritated wounds (6). Carrel also claimed that the addition of live or dead leukocytes to tissue culture media increased the rate of outgrowth of fibroblasts from tissue explants due to the presence of "trephones" in the leukocytes (8). Since the work of Carrel, numerous other investigators have presented evidence that inflammation promotes the repair process (4, 9-12).

The following study was designed to investigate the role of the neutrophilic leukocyte in wound repair by examining the healing process in the absence of these cells. Neutrophils were eliminated from the wounds by inducing a neutropenia with heterologous antineutrophil serum (ANS). The neutropenia was maintained by daily administration of antineutrophil serum over a 10

\footnotetext{
${ }^{1}$ Abbreviations used in this paper: ANS, antineutrophil serum; HBSS, Hank's balanced salt solution; NRS, normal rabbit serum; PBS, phosphate-buffered saline; PMN, polymorphonuclear neutrophilic leukocytes.
} 
day period of healing. The extent of connective tissue repair and the efficiency of debridement in neutropenic wounds compared with controls were of particular interest.

\section{METHODS}

Experimental animals. Strain 13 guinea pigs were used both for obtaining neutrophils for antigen in the preparation of ANS and for the wound healing studies.

Preparation of antineutrophil serum. Peritoneal exudates were obtained from 400-500-g guinea pigs $18 \mathrm{hr}$ after intraperitoneal administration of $40 \mathrm{ml}$ of sterile $3 \%$ proteose peptone (Difco Labs, Detroit, Mich.), as described by Humphrey (13). The peritoneal cavity was opened surgically using ether anesthesia, and the exudate was carefully pipetted from the peritoneal cavity into heparinized $0.9 \%$ saline (10 $\mathrm{U} / \mathrm{ml}$ ). Differential counts were performed on Giemsastained smears of undiluted exudate. The exudates usually contained $93-97 \%$ neutrophils, 3-7\% mononuclear leukocytes, and small numbers of red cells and mesothelial cells. Exudates containing less than $90 \%$ neutrophils were discarded.

The exudate cells were sedimented by gentle centrifuging at $100 \mathrm{~g}$, washed briefly in $0.5 \%$ sodium citrate to lyse erythrocytes (14), then washed two more times in normal saline. The cells were counted and suspended in saline at a concentration of $2 \times 10^{8} / \mathrm{ml}$. Three adult, male New Zealand rabbits were immunized three times at 1-wk intervals with $2 \mathrm{ml}$ of fresh cell suspension $\left(4 \times 10^{8}\right.$ total cells $)$ each time. The cell suspensions were administered subcutaneously at different sites on the shaved backs of the rabbits. At the first immunization, $1 \mathrm{ml}$ of Freund's complete adjuvant (Difco Labs) was mixed with the cell suepension before injection.

1 wk after the final immunization, the rabbits were sacrificed by cardiac puncture. The antiserum was collected, pooled, and inactivated at $56^{\circ} \mathrm{C}$ for $30 \mathrm{~min}$. After titration and absorption, ANS was sterilized by filtration, dispensed into 2 -ml vials, and stored at $-20^{\circ} \mathrm{C}$ until used.

Normal rabbit serum (NRS) for control experiments was collected from untreated rabbits from the same stock used for ANS preparation. Control serum was handled in a fashion identical with that of the ANS.

Titration of ANS. Leuko-agglutination titers of ANS against neutrophils and lymphocytes were determined as described previously (15). Neutrophils for agglutination titers were obtained from peptone-stimulated peritoneal exudates. Lymphocytes were collected from minced lymph nodes which were pressed through a stainless steel 60 mesh wire screen into Hank's balanced salt solution (HBSS). All leukocytes used for titration were washed three times in $0.01 \mathrm{M}$ phosphate-buffered saline (PBS) before use. Agglutination was assessed by low-power phase-contrast microscopy of the cell

TABLE I

In Vitro Characterization of $A N S$

\begin{tabular}{|c|c|c|c|}
\hline \multicolumn{3}{|c|}{ Agglutination titers* } & \multirow{2}{*}{$\begin{array}{c}\text { Cytotoxic } \\
\text { titer }\end{array}$} \\
\hline PMN & LYMPH & $\mathrm{RBC}$ & \\
\hline $1: 320$ & $1: 80$ & $1: 40$ & $1: 2500$ \\
\hline
\end{tabular}

* Before any absorption.

$\ddagger$ Titer is highest serum dilution at which no less than $50 \%$ of neutrophils were stained with trypan blue. suspension $1 \mathrm{hr}$ after addition of the antiserum. The titer was determined as the highest dilution of ANS containing distinctly larger clumps of leukocytes than the NRS control tubes, which usually contained clumps of three to four leukocytes as well as numerous free cells at all dilutions.

The cytotoxic titer of ANS against neutrophils was determined by the technique of Miller and Wilson (16), using exudate neutrophils in HBSS. The per cent of neutrophils staining with trypan blue was determined at each dilution of ANS; the cytotoxic titer was taken as the highest dilution of ANS at which not less than $50 \%$ of the neutrophils were stained with the dye. Control tubes of dilutions of NRS usually contained $8-10 \%$ stained cells.

Hemagglutination titers were determined for ANS and control sera using fresh, thrice-washed guinea pig erythrocytes obtained by cardiac puncture. The tubes containing diluted serum and erythrocytes were allowed to stand overnight before reading.

Absorption of $A N S$. Antibody to a single component of guinea pig serum was present in ANS as determined by double-diffusion analysis in agar gels (Immunoplate, Hyland Laboratories, Los Angeles, Calif.). Thus, ANS was absorbed with normal guinea pig serum $(0.05 \mathrm{ml} / \mathrm{ml}$ of ANS) for $1 \mathrm{hr}$ at room temperature with shaking. The serum was then centrifuged at $30,000 \mathrm{~g}$ for $30 \mathrm{~min}$ at $4^{\circ} \mathrm{C}$ and the supernate was collected. The absorbed serum gave no precipitin line in double-diffusion plates.

ANS was absorbed with guinea pig erythrocytes obtained by cardiac puncture and washed three times in PBS. Two absorptions for $1 \mathrm{hr}$ each at room temperature using $5-\mathrm{ml}$ packed red cells/25 ml ANS was sufficient to remove hemagglutinating antibodies.

A $30 \mathrm{ml}$ portion of ANS was absorbed for $1 \mathrm{hr}$ with $5 \times 10^{8}$ lymphocytes from cervical, axillary, and mesenteric nodes.

Experimental design and wounding. Guinea pigs weighing 250-300 $\mathrm{g}$ were utilized because less antiserum was necessary to achieve the desired neutropenia. 5 animals served as controls and received NRS, while 10 animals were injected with ANS. ANS or NRS injections were begun 24 $\mathrm{hr}$ before wounding. Serum was administered intraperitoneally with a 26 gauge needle at an initial dosage of 3 $\mathrm{ml} / \mathrm{kg}$ per day. The serum was divided into two equal doses and administered twice daily at 12 -hr intervals at the same times each day. Blood samples were taken before the first daily injection by making a small incision at the periphery of the ear after topical application of toluene for vasodilation. Total blood counts were determined in a hemocytometer. Differential counts were performed as "wet counts" in the hemocytometer and on Wright's-stained blood smears. Occasional blood samples were collected in heparinized capillary tubes for microhematocrits.

ANS and NRS administration was continued throughout the 10 day course of wound healing. After the first 3 days it became necessary to increase the total daily dose of serum by $0.1 \mathrm{ml}$ in order to maintain the desired neutropenia.

$24 \mathrm{hr}$ after the initiation of ANS or NRS injections, the animals were lightly anesthetized with ether and wounded with a series of six linear scalpel incisions on their backs. The hair was shaved from the backs of the guinea pigs and the skin was carefully cleansed with $70 \%$ ethanol. Each wound was approximately $1.5 \mathrm{~cm}$ long and extended through the panniculus carnosus.

Each guinea pig was caged separately throughout the course of the experiments. They had access to a standard guinea pig pellet diet containing ascorbic acid (Ralston 
Purina, St. Louis, Mo.) and water ad lib. With the animals under light ether anesthesia, wounds were removed at 1,2 , $3,5,7$, and 10 days by excising an ellipse of skin containing the initial incision. The biopsy site was closed with wound clips. At the termination of the experiment, the animals were sacrificed and portions of liver, spleen, lymph node, bone marrow, and kidney were prepared for microscopy.

\section{Preparation of wounds for microscopy}

Light microscopy. Immediately after its removal, the ellipse of skin containing the wound was halved transversely. One-half of the wound was fixed in $10 \%$ neutralbuffered formalin and embedded in paraffin. Wound sections were stained with hematoxylin and eosin, phosphotungstic acid-hematoxylin, van Gieson's, and Gomori's trichrome stains.

Electron microscopy. The remaining half of each wound was immersed in a pool of fixative on dental wax. Using razor blades, small slices of tissue, approximately 1-2 mm, were cut so that each contained a cross-section of the wound from an area in the central portion of the original incision.

Wound blocks were fixed one of two ways. Most were fixed for $5 \mathrm{hr}$ at room temperature in a one-half strength modification of Karnovsky's glutaraldehyde-paraformaldehyde mixture (17), washed overnight at $4^{\circ} \mathrm{C}$ in $0.1 \mathrm{M}$ cacodylate buffer containing $7.5 \%$ sucrose, and postfixed for $1 \mathrm{hr}$ at $4^{\circ} \mathrm{C}$ in buffered $2 \% \mathrm{OsO}_{4}$. Other blocks were fixed initially in buffered $2 \% \mathrm{OsO}_{4}$ at $4^{\circ} \mathrm{C}$ for $1-1 \frac{1}{2} \mathrm{hr}$, washed briefly in $0.2 \mathrm{~m}$ s-collidine buffer, then fixed $1 \mathrm{hr}$ at room temperature in $3 \%$ paraformaldehyde in $0.2 \mathrm{M}$ phosphate buffer ( $\mathrm{pH}$ 7.4). Before dehydration, many of the blocks were stained with $0.5 \%$ uranyl acetate in $0.2 \mathrm{~m}$ s-collidine buffer ( $\mathrm{pH}$ 6.1) at room temperature for $1 \mathrm{hr}$. The blocks were embedded in Epon, and oriented so that cross-sections of the wounds would be obtained upon sectioning.

$1-\mu$ sections were routinely stained with azure II methylene blue; others were stained with basic fuchsin and alkalinized methylene blue (18). Utilizing the $1-\mu$ sections for orientation, the blocks were further trimmed to the desired area for thin sectioning. The thin sections were placed on carboncoated grids, stained with uranyl acetate and lead tartrate, and examined in an AEI-6B electron microscope.

Morphometric technique for light microscopy. A stereological technique was utilized to provide a quantitative determination of the relative volume of various components present in 24-hr neutropenic and control wounds (19). 1- $\mu$ Epon sections stained with basic fuchsin-methylene blue were counted in the Zeiss photoscope (Carl Zeiss, Inc., New York) at high, dry magnification $(\times 1600)$ with a $10 \mathrm{~mm}$ square net reticule lined in $0.5-\mathrm{mm}$ intervals (American Optical Corp., Buffalo, N. Y.).

Structures that were recorded included: neutrophils, monocytes (macrophages and lymphocytes), fibrin, red blood cells, "space," and others (includes hairs, fat, granulocytes other than neutrophils, occasional collagen fibers, unidentifiable cells, etc.). The category listed as "space," that area not containing discrete structures, is thought to represent exudative fluid including plasma proteins and is also thought to arise, in part, from tissue processing artifact. Unidentifiable cells represented less than $1 \%$ of the total counts.

Sections of 24-hr wounds were counted from three NRScontrol animals and from three ANS-treated animals. The sections from each guinea pig were from different blocks within a single wound and were thus physically separated by at least $1-2 \mathrm{~mm}$ in vivo. The mean counts from neutropenic and control wounds were compared statistically within each category with a $t$ test.

In order to test the accuracy of the counting technique, counts were performed utilizing serial $1-\mu$ sections from a single wound block. Four of these sections selected at approximately $20-\mu$ intervals were stained and counted. Good internal agreement was obtained among these four sections, indicating that a high degree of accuracy and reproducibility could be expected. Duplicate counts of the same section on different days gave close agreement.

\section{RESULTS}

\section{Hematologic and systemic response to prolonged ANS or NRS administration}

The absolute peripheral white blood cell counts and weights for both the neutropenic and control guinea pigs over the 11 days during which ANS or NRS were administered are plotted in Fig. 1. Table II lists the daily absolute peripheral leukocyte counts for a typical guinea pig receiving ANS and another receiving NRS.

Neutropenic animals. In guinea pigs given ANS, circulating neutrophils remained low throughout the 11 days of antiserum administration (Fig. 1). At the time of wounding, $24 \mathrm{hr}$ after initiation of ANS administration, neutrophils were at extremely low levels $\left(<100\right.$ cells $\left./ \mathrm{mm}^{3}\right)$. In some of the animals, neutrophils remained below $150 / \mathrm{mm}^{3}$ during the course of the experiment. Most of the circulating neutrophils seen during ANS treatment were immature (band) forms.

Lymphocytes also showed a severe initial decrease in the neutropenic animals to approximately $70 \%$ below starting levels after $24 \mathrm{hr}$ of antiserum administration. In spite of continued ANS administration, the lymphocyte counts increased rapidly until day 7 , when the mean count was approximately $20 \%$ below the starting level. Thereafter the lymphocyte count tended to decrease gradually until the termination of the experiment at 11 days. The decrease in lymphocytes after ANS was not due simply to a cross-reactivity of ANS with these cells, since administration of lymphocyteabsorbed ANS produced the same peripheral lymphocyte response both in guinea pigs used for woundhealing studies and in a series of guinea pigs which received only a single dose of ANS in a separate study (15). Other investigators $(13,14)$ have also noted an unexplained fall in peripheral lymphocytes after ANS administration.

Monocyte counts fell approximately 50\% within 24 $\mathrm{hr}$ after the initial injection of ANS. After the 2nd day the number of monocytes generally returned to starting levels, and were similar to the controls during the remainder of the experiment (Fig. 1). (The monocyte counts in the experimental animals overlapped the neutrophil counts and hence are not shown in Fig. 1.) 
7 days after beginning ANS administration, absolute increases were noted both in eosinophil and in basophil counts. The increase in basophils was particularly dramatic since extremely few were present before beginning the ANS injections. In some of the animals there were over 400 basophils $/ \mathrm{mm}^{3}$ between the 7 th and 11th days of ANS administration. Examination of marrow smears at 11 days revealed an increase in the per cent of basophils in guinea pigs receiving ANS.

In spite of their severe neutropenia, most of the guinea pigs injected with ANS appeared healthy, and their weight remained stable over the 11 days (Fig. 1). Three of the neutropenic animals died during the 3rd to the 5th day of the experiment. Autopsies revealed the presence of infection in the peritoneal cavity. Two of the neutropenic guinea pigs were sacrificed on the 7 th day of the wound healing due to lethargy and weight loss which indicated the possible onset of infection.

Control animals. In guinea pigs injected with NRS, the circulating neutrophil counts increased rapidly until the 5th day, at which time the mean number of neutrophils was approximately four times the starting level (Fig. 1). By the end of the experiment the neutrophil counts had returned to nearly original values. The reason for the significant rise in neutrophils was not apparent, but the neutrophilia is probably related in part to the systemic stress of initial wounding and sequential biopsy and blood sampling procedures. Even though daily blood sampling from the ear resulted in the removal of only $0.25-0.50 \mathrm{ml}$ of blood, in an animal the size of the guinea pig this could result in stimulation of the marrow.

After an initial slight decrease for 2 days, the num-
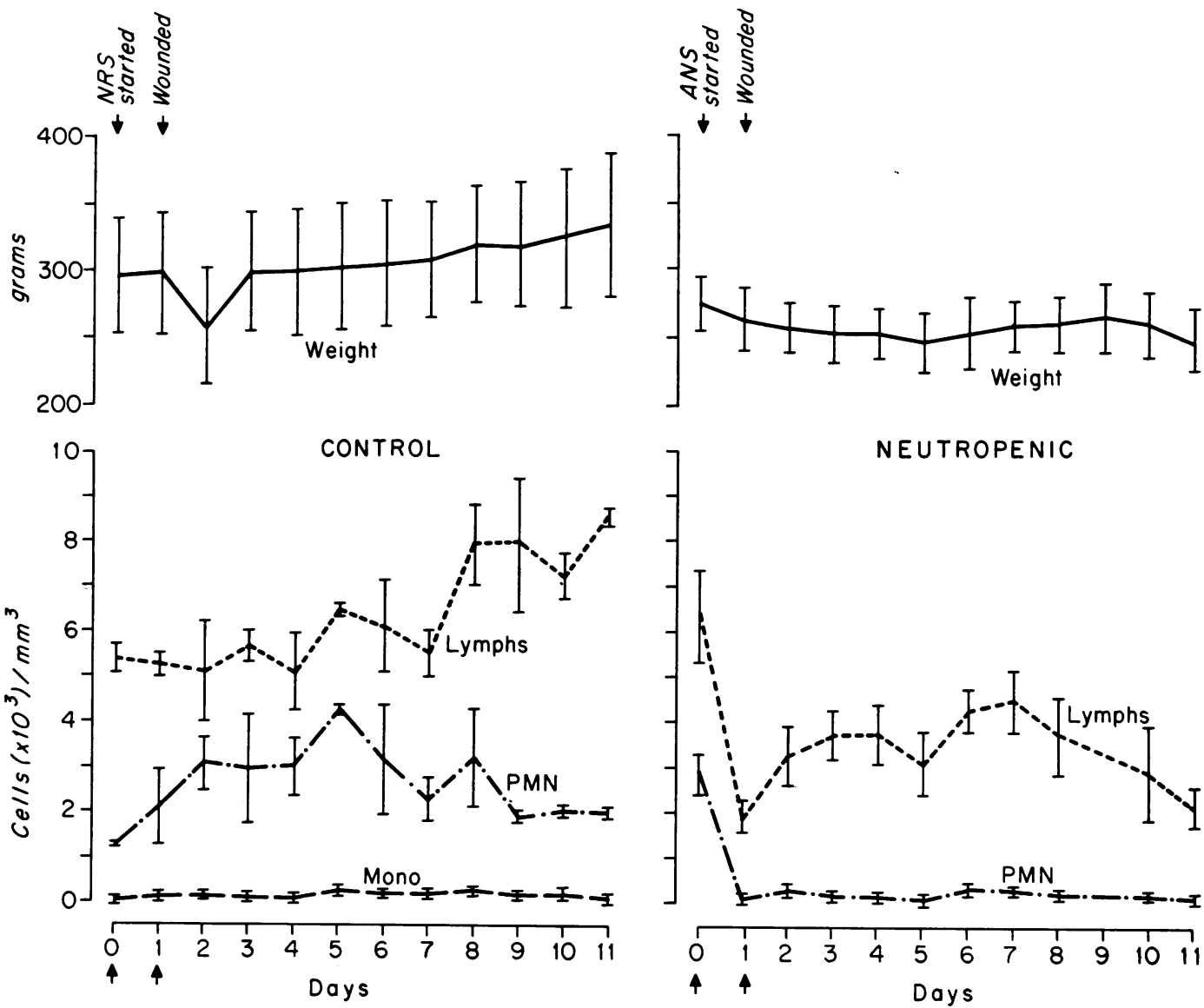

FIGURE 1 These graphs illustrate the absolute peripheral white blood cell counts and the weights for the control and neutropenic guinea pigs over the 11 day period during which NRS or ANS were administered on a twice daily basis. Serum injections were started on day " 0 ," $24 \mathrm{hr}$ before wounding. The monocyte counts for the neutropenic animals, although not plotted here due to overlap with the PMN curve, were similar to those for the control group with the exception that a decrease of approximately $50 \%$ was observed at day 1 . The vertical bars represent $\pm 1 \mathrm{SE}$. 
TABLE II

Typical Daily Blool Counts for Individual Guinea Pigs Injected Twice Daily with ANS or NRS throughout a 10 Day Course of Wound Healing

\begin{tabular}{|c|c|c|c|c|c|c|c|c|c|c|c|c|c|}
\hline \multirow[b]{2}{*}{ Day } & \multirow[b]{2}{*}{ Procedure } & \multicolumn{6}{|c|}{$\begin{array}{l}\text { Antineutrophil serum } \\
\text { (Guinea pig 70-17) }\end{array}$} & \multicolumn{6}{|c|}{$\begin{array}{l}\text { Normal rabbit serum } \\
\text { (Guinea pig } 69-34 \text { ) }\end{array}$} \\
\hline & & Total & PMN & Eos. & Baso. & Lymph. & Mono. & Total & PMN & Eos. & Baso. & Lymph. & Mono. \\
\hline 0 & ANS or NRS started & 6360 & 2226 & 64 & $\mathbf{0}$ & 3980 & 90 & 6,490 & 1272 & 130 & 0 & 5056 & 32 \\
\hline 1 & Wounded & 1200 & 24 & 36 & 0 & 1092 & 48 & 6,850 & 1250 & 0 & 0 & 5496 & 104 \\
\hline 2 & 1 day wound removed & 2280 & 114 & 46 & 182 & 1870 & 68 & 6,650 & 2505 & 67 & 67 & 3844 & 167 \\
\hline 3 & 2 day wound removed & 3680 & 110 & 74 & $\mathbf{0}$ & 3349 & 147 & 7,260 & 1742 & 218 & 73 & 5082 & 145 \\
\hline 4 & 3 day wound removed & 2720 & 27 & 0 & 27 & 2611 & 55 & 6,780 & 2373 & 136 & 68 & 4101 & 102 \\
\hline 5 & & 3440 & 0 & 34 & 34 & 3302 & 68 & 11,220 & 4208 & 112 & 0 & 6507 & 393 \\
\hline 6 & 5 day wound removed & 4800 & 280 & 192 & 432 & 3792 & 104 & 7,340 & 1945 & 73 & $\mathbf{0}$ & 5138 & 184 \\
\hline 7 & & 7120 & 285 & 285 & 142 & 6337 & 71 & 9,000 & 2745 & 180 & 0 & 5895 & 180 \\
\hline 8 & 7 day wound removed & 6080 & 243 & 304 & 182 & 5107 & 243 & 11,240 & 2136 & 225 & 0 & 8598 & 281 \\
\hline 9 & & 5740 & 220 & 180 & 265 & 4883 & 192 & 11,720 & 1992 & 703 & 352 & 8497 & 176 \\
\hline 10 & & 4940 & 290 & 50 & 395 & 3952 & 253 & 9,300 & 2139 & 651 & 186 & 6231 & 93 \\
\hline 11 & $\begin{array}{l}10 \text { day wound } \\
\text { removed, sacrificed }\end{array}$ & 4340 & 122 & 43 & 174 & 3914 & 87 & 10,560 & 1901 & 1056 & 422 & 7023 & 158 \\
\hline
\end{tabular}

ber of circulating lymphocytes increased gradually over the next 9 days. At the end of the experiment peripheral lymphocyte counts were approximately $50 \%$ above original levels, possibly reflecting immunization of the animals to NRS. As in the neutropenic guinea pigs, the eosinophil and basophil counts demonstrated a dra-

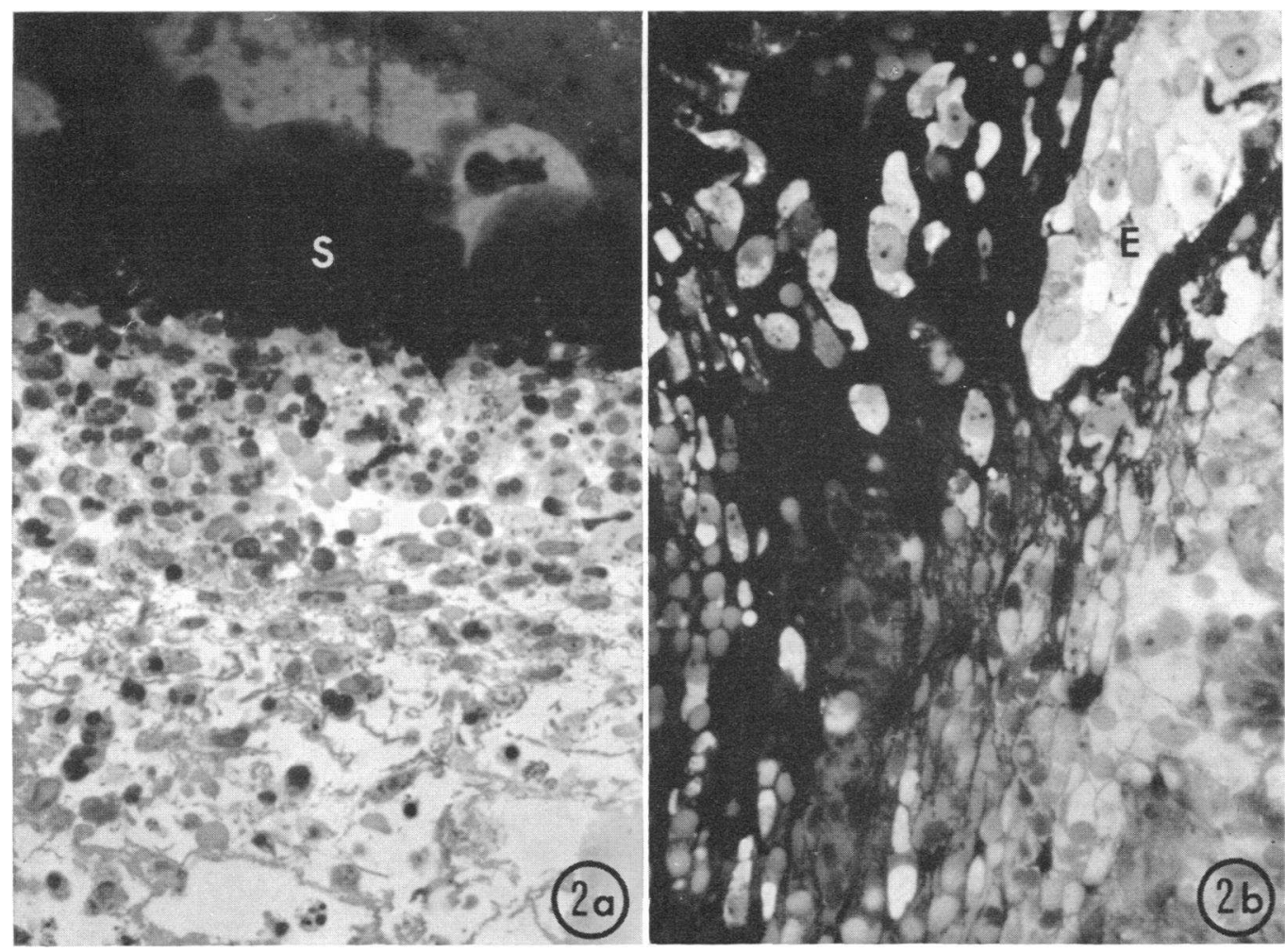

Figure 2 (a) Light micrograph from an area just inferior to the base of the scab (S) of a 24 hour wound from a control animal. Note the dense accumulation of leukocytes, primarily neutrophils, beneath the scab. Mononuclear leukocytes and strands of fibrin are also evident. Magnification $\times 520$. (b) Light micrograph from an area at the base of the scab at the margin of a 24 hour wound from a neutropenic animal. The margin of migrating epithelium (E) is visible. Mononuclear leukocytes and erythrocytes are prominent; no neutrophils are present. Magnification $\times 520$. 

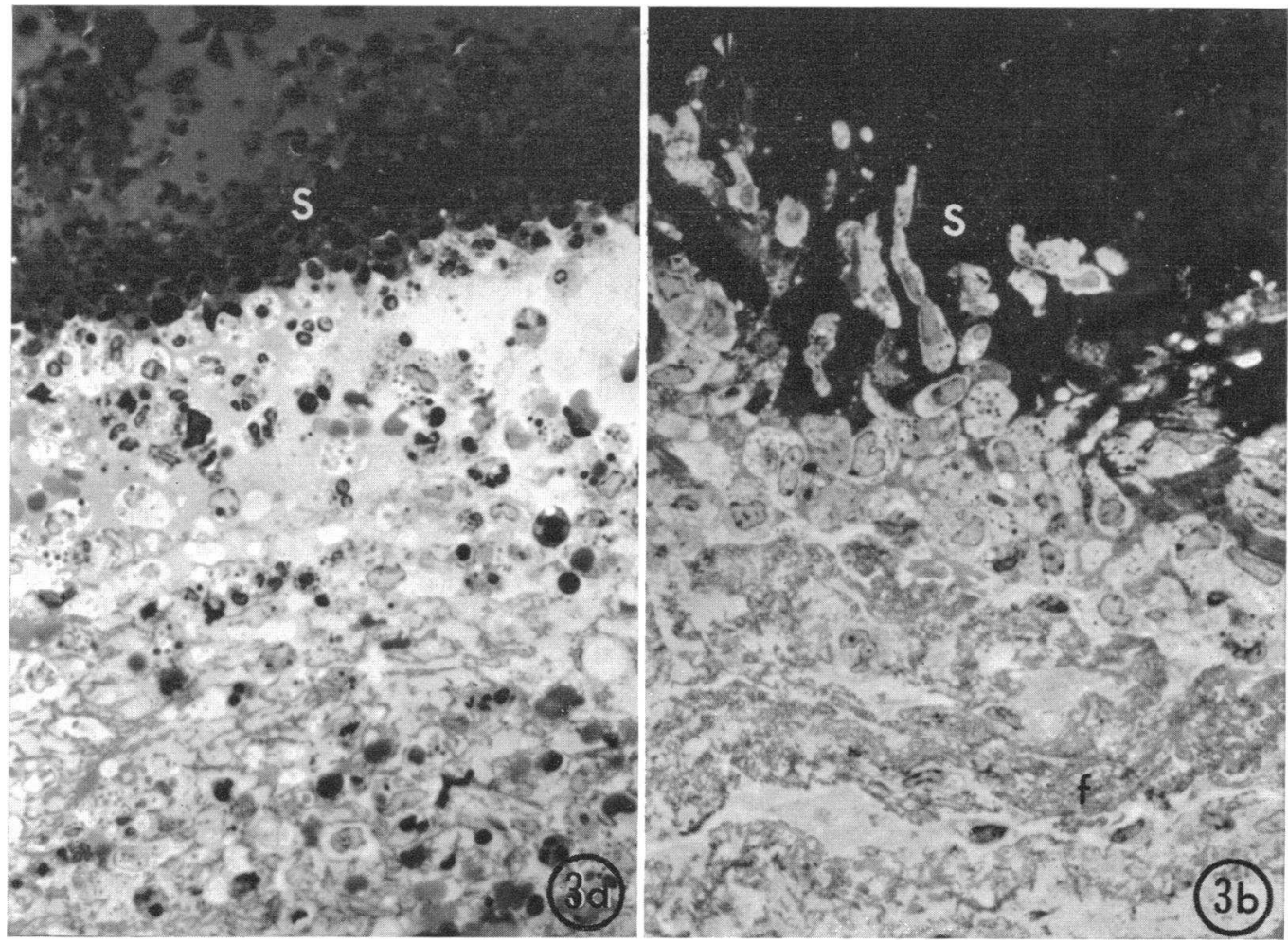

FIgURE 3 (a) This micrograph illustrates a portion of wound exudate and the base of the scab (S) of a 48 hour wound from a control animal. Many neutrophils and mononuclear cells are present in the exudate at this time. A distinct accumulation of leukocytes is visible in the base of the scab, forming a characteristic layer of cells demarcating the inferior border of the scab. Magnification $\times 560$. (b) This micrograph illustrates a portion of a neutropenic wound at $48 \mathrm{hr}$ in an area comparable with that seen in the control wound in Fig. $3 a$. Numerous mononuclear phagocytes are present in the exudate and at the base of the scab (s), but neutrophils are absent. Note the fibrin strands (f) in the exudate. Magnification $\times 560$.

matic increase beginning on about the 7 th to 8 th day of NRS injection.

\section{Light microscopy of wound repair}

Inflammatory phase-control wounds. Wounding in both groups of animals resulted in hemorrhage and formation of a fibrin clot. Beneath the resultant scab of 24-hr control wounds the interstices of the clot were filled with numerous erythrocytes, neutrophils, monocytes, and spaces presumably occupied by extravasated serum (Fig. $2 a$ ). At this time, some of the monocytes had already begun ingesting debris and some of them contained ingested neutrophils.

When the early wounds of the two groups of animals were compared, the difference in cellularity was particularly apparent in the region at the base of the scab. In 24 and 48-hr control wounds, numerous leukocytes characteristically were present in this region (Figs. $2 a$ and $3 a$ ). Many of these leukocytes had become incorporated within the lower portion of the scab, forming a distinct accumulation of cells demarcating its inferior border. Neutrophils were the predominant cell type beneath the scabs of control wounds and, in agreement with previous observations (1), neutrophils were the predominant inflammatory cells throughout the 24-hr control wounds. By 2-3 days, the number of neutrophils had decreased in relation to the number of monocytes. Abundant fibrin and red cells remained at 2 and 3 days, and numerous macrophages with phagocytic vacuoles were present throughout the wounds.

Inflammatory phase-neutropenic wounds. The most striking difference between neutropenic and control wounds at 24 and $48 \mathrm{hr}$ was the lack of neutrophils in the ANS-treated animals. At low magnification this difference was apparent as a greatly decreased over-all cellularity (Figs. $2 b, 3 b$ ).

In the 24 and $48-\mathrm{hr}$ neutropenic wounds, the characteristic layer of neutrophils present beneath the scabs in control wounds was absent. Monocytes were present throughout the exudates of the neutropenic wounds. 
Most of them were phagocytic, and many contained large cytoplasmic vacuoles within which red cells, fibrin, and unidentifiable debris could be observed, par- ticularly by the 3 rd day. The number of monocytes and their phagocytic activity appeared to be the same in the control and neutropenic wounds.

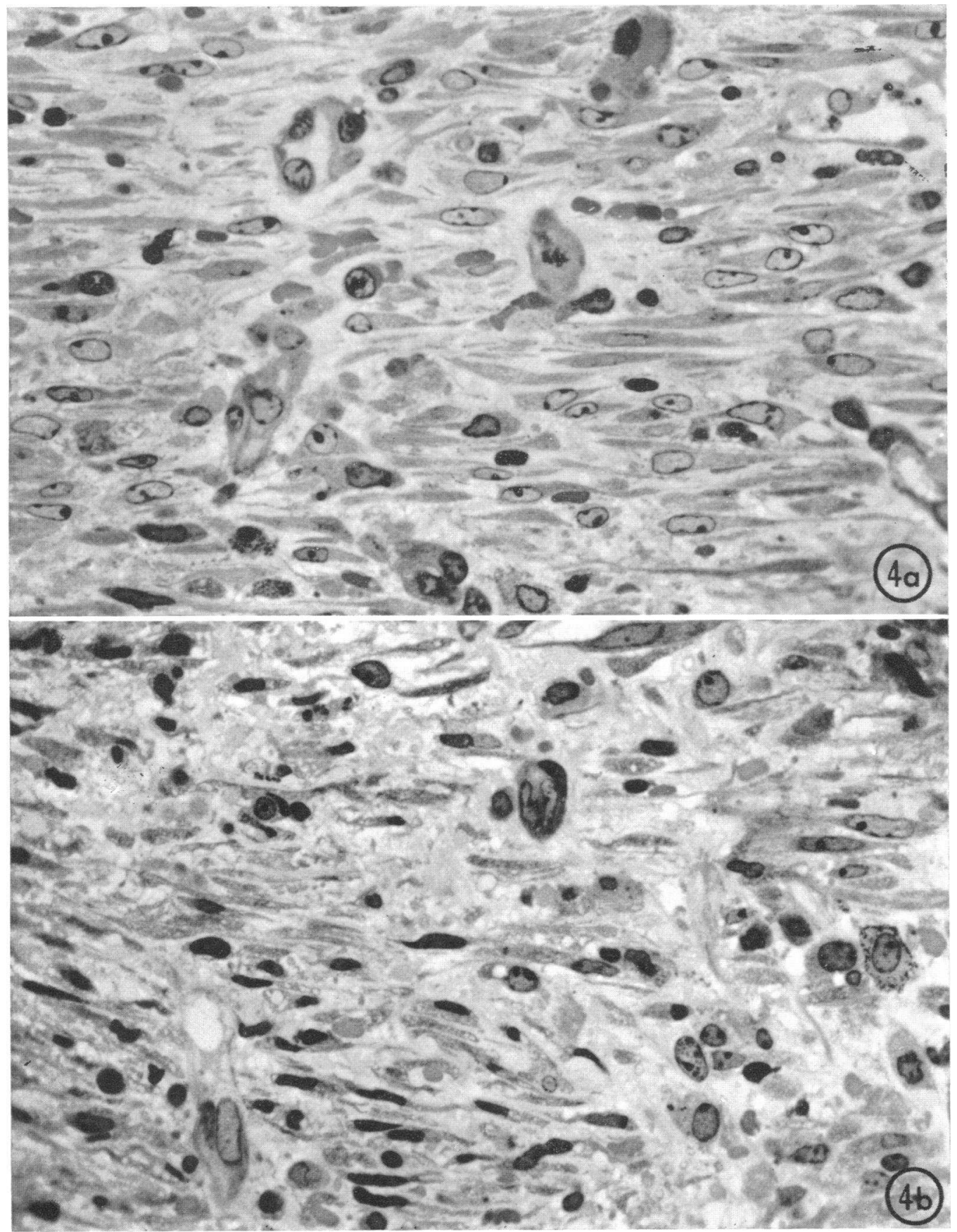

FIGURE 4 Light micrographs of 7-day wounds in an area just beneath the regenerated epithelium. (a) Control guinea pig. Numerous stellate and spindle-shaped fibroblasts, small vessels, and occasional macrophages are present. By 7 days of healing, a moderate amount of intercellular collagen is present. (b) Neutropenic guinea pig. The extent of repair in the experimental animals is identical with that in the controls at this time. 1- $\mu$ sections, both micrographs $\times 600$. 


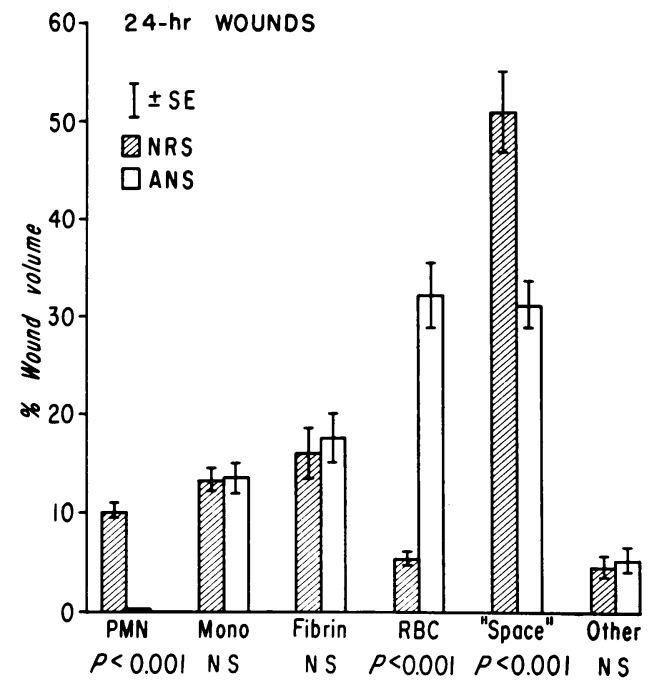

FIGLRE 5 Histogram showing a comparison of the per cent of the total wound volume occupied by the various major components in 24-hr control and neutropenic wounds. This data was obtained with 1- $\mu$ Epon sections using a histometric counting method. With a grid in the microscope ocular, the structures beneath the grid intersections were counted at high magnification over the entire wound area, from the base of the scab to the superior border of the panniculus carnosus. The category listed as "space" was histologically empty and was presumed to represent space occupied by exudate. Mono, mononuclear leukocytes.

Although there was wide variation in the pattern of deposition of fibrin, there were no discernible differences in fibrin content or distribution when 1,2 , or 3 day neutropenic and control wounds were compared.

Fibrosis-neutropenic vs. controls. Between the 5th and 7 th days of healing, macrophages and fibroblasts were the predominant cell types in the wounds (Fig. 4). By this time there were no observable differences in the progress of repair in the wounds from ANS or NRS-treated animals. Fibroblasts could be identified in $1-\mu$ sections as elongated, spindle-shaped or tripolar cells with round to oval nuclei usually containing a single nucleolus. In contrast, macrophages were larger, more irregularly-shaped cells, often with indistinct cytoplasmic borders, and their nuclei were characteristically oval or reniform in shape. At 5-7 days, many of the macrophages in the granulation tissue contained ingested debris or red cells. New vessels in the granulation tissue could usually be identified by the presence of erythrocytes within their lumens. The epithelium had regenerated over the wounds within 5-7 days.

Beginning at the 7 th day, frequent basophils were observed within the wounds from both groups of animals. Basophils were also present in large numbers in the dermis adjacent to the wounds. This presumably reflected increased emigration from small vessels adja- cent to and within the wound at a time corresponding to the onset of blood basophilia.

The extent of healing in 7 and 10-day wounds from control and experimental animals was identical in terms of the number of fibroblasts and the amount of collagen observed in tissue sections (Fig. 4). No differences were observed in the 10-day neutropenic and control wound sections stained for collagen.

Quantitation of components in 24-hr wounds. The results of histometric counts on 1- $\mu$ Epon sections of 24-hr neutropenic and control wounds are shown in Fig. 5.

In control wounds, neutrophils occupied approximately $10 \%$ of the total volume at $24 \mathrm{hr}$, in contrast to the neutropenic wounds in which neutrophils occupied only a small volume $(0.2 \%)$.

No significant difference was observed in wound volume occupied by monocytes in the two groups. Although monocytes form a numerically smaller population of cells as compared with neutrophils in 24-hr wounds, the portion of total wound volume occupied by monocytes $(13.4 \%$ in controls, $13.5 \%$ in neutropenic) was somewhat greater than that of the neutrophils $(10.1 \%$ in controls $)$. This is due to the larger size of the monocytes, especially those containing ingested debris.

There were significant differences in wound volume occupied by red blood cells and "space" in the control and neutropenic wounds. Red cells occupied almost six times the wound volume in 24-hr neutropenic wounds as compared with controls. This difference in distribution of red cells in the two sets of wound was apparent histologically, but the extent of the difference was not appreciated before quantitation.

\section{Electron microscopy of wound repair}

Ultrastructural examination of 1,2, and 3-day wounds from ANS-treated animals confirmed that neutrophils were essentially absent from these wounds. The few neutrophils that were present were band cells or metamyelocytes containing varying amounts of rough endoplasmic reticulum and Golgi apparatus as well as nonsegmented or partially-segmented nuclei, features characteristic of granulocytes usually found only in marrow (20).

Fig. 6 illustrates a characteristic area containing numerous mature neutrophils in the exudate of a $24 \mathrm{hr}$ control wound. The neutrophils showed little evidence of phagocytic activity in these aseptic wounds. Some of the neutrophils in early control wounds demonstrated degenerative changes while other had undergone lysis, releasing their granules into the extracellular space.

In 2 and 3-day neutropenic and control wounds, most of the monocytes had become phagocytic and contained 


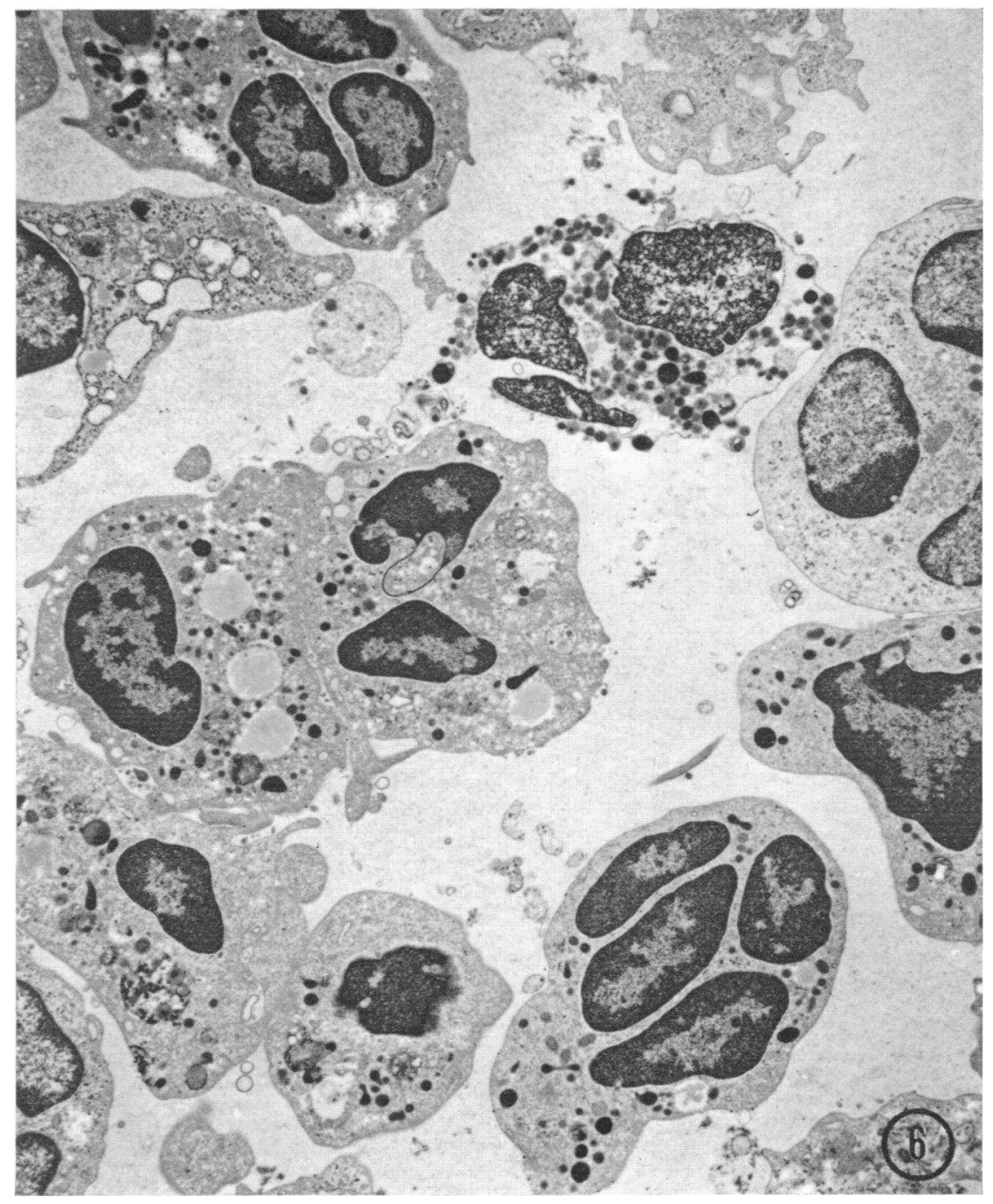

FIGURE 6 This electron micrograph illustrates a portion of exudate in the center of a $24 \mathrm{hr}$ wound from a control animal. There is a preponderance of neutrophilic leukocytes, most of which are intact. A single lysed neutrophil missing a portion of plasma membrane is evident. Some of the granules from this cell have been released into the extracellular space. Several of the neutrophils contain cytoplasmic deposits of amorphous or granular debris presumably indicative either of limited phagocytic activity or of degenerative change. The intercellular space is occupied by exudate containing serum proteins, visible as slightly granular background material. Magnification $\times 5700$.

erythrocytes, myelin figures, fibrin, and other wound debris. In this transition these cells increased in size and acquired greater amounts of rough endoplasmic reticulum with moderate numbers of attached ribosomes, increased numbers of smooth membrane-bounded vacuoles, and irregular borders possessing small, microvillous projections.

As early as 3 days after wounding, immature fibroblasts could be identified within the wound exudate
(Fig. 7). As these cells matured, they acquired the characteristic ultrastructural features of actively synthetic fibroblasts, as described previously $(2,21)$. By 5 days, small collagen fibrils were observable by electron microscopy within the extracellular spaces of both neutropenic and control wounds. In 7-day wounds the amount of collagen had increased considerably. At this time the cell population consisted primarily of fibroblasts, macrophages, endothelial cells, and occasional 
basophils. There were no differences in the ultrastructural appearances of the 7 and 10-day wounds from control or neutropenic guinea pigs (Fig. 8).

\section{DISCUSSION}

In an extensive review of wound healing in 1936, Arey (4) discussed numerous investigations that presented evidence for "wound hormones" or other hypothetical stimulatory agents believed to initiate or promote local cellular activities in wounds. Most early investigators thought that either the injured tissue or the infiltrating leukocytes released some type of growth-promoting substance at the site of injury. Experiments, claiming to improve healing, following the topical application of leukocytes or embryo juice were cited as evidence for growthpromoting substances. Arey also believed that enzymes

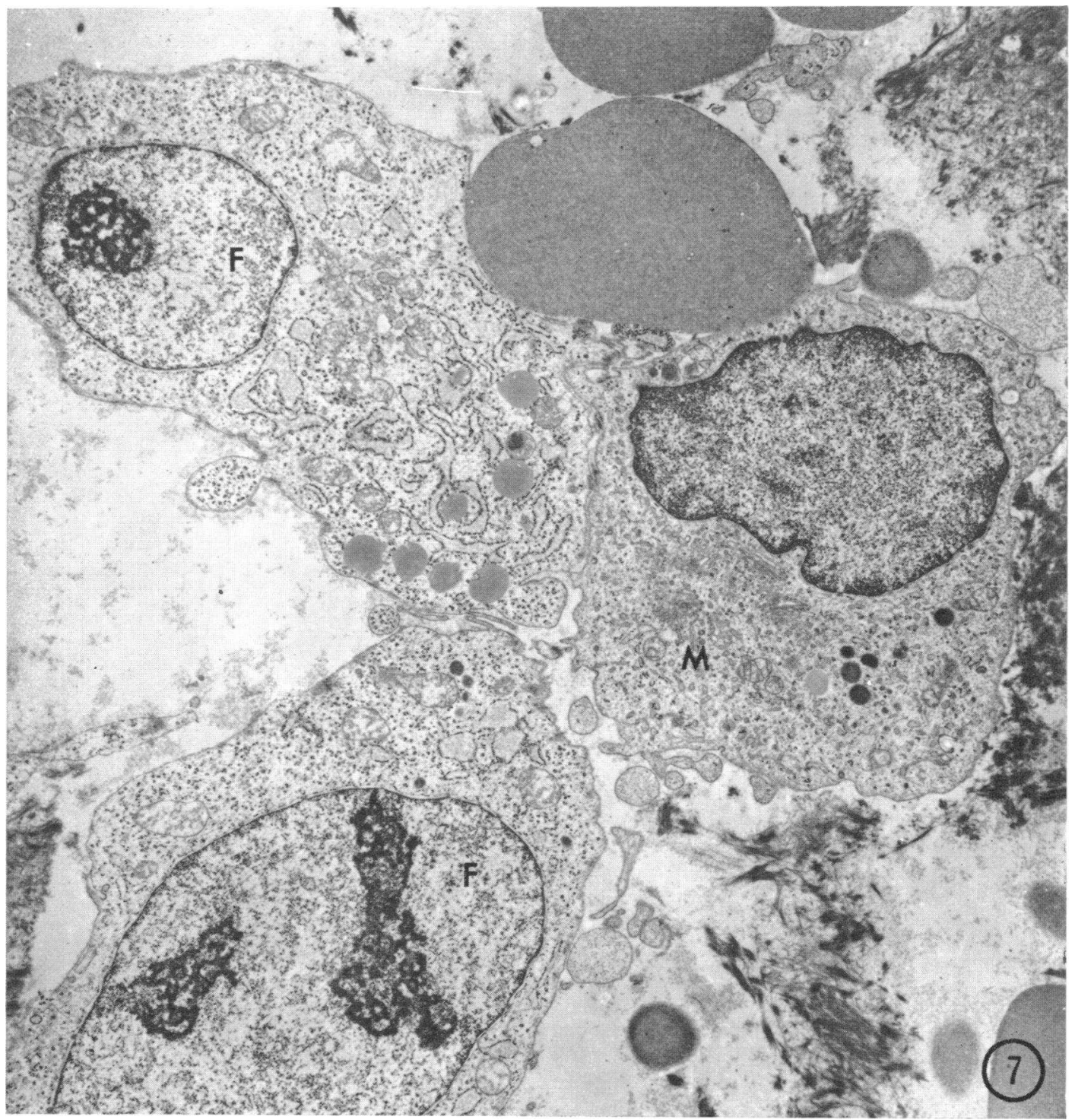

FIGURE 7 This electron micrograph illustrates the characteristic ultrastructural features of young fibroblasts $(F)$ and a mononuclear phagocyte $(M)$ within a 3 day neutropenic wound. These two cell types appear similar by light microscopy but may generally be differentiated by electron microscopy. The immature fibroblasts $(F)$ contain numerous free cytoplasmic ribosomes as well as a developing rough endoplasmic reticulum. The oval fibroblast nuclei contain large nucleoli. Several lipid deposits can be seen within one of the fibroblasts. The mononuclear phagocyte in contrast, contains a large, perinuclear Golgi complex, a small amount of poorly developed rough endoplasmic reticulum, and several dense bodies. Magnification $\times 5400$. 


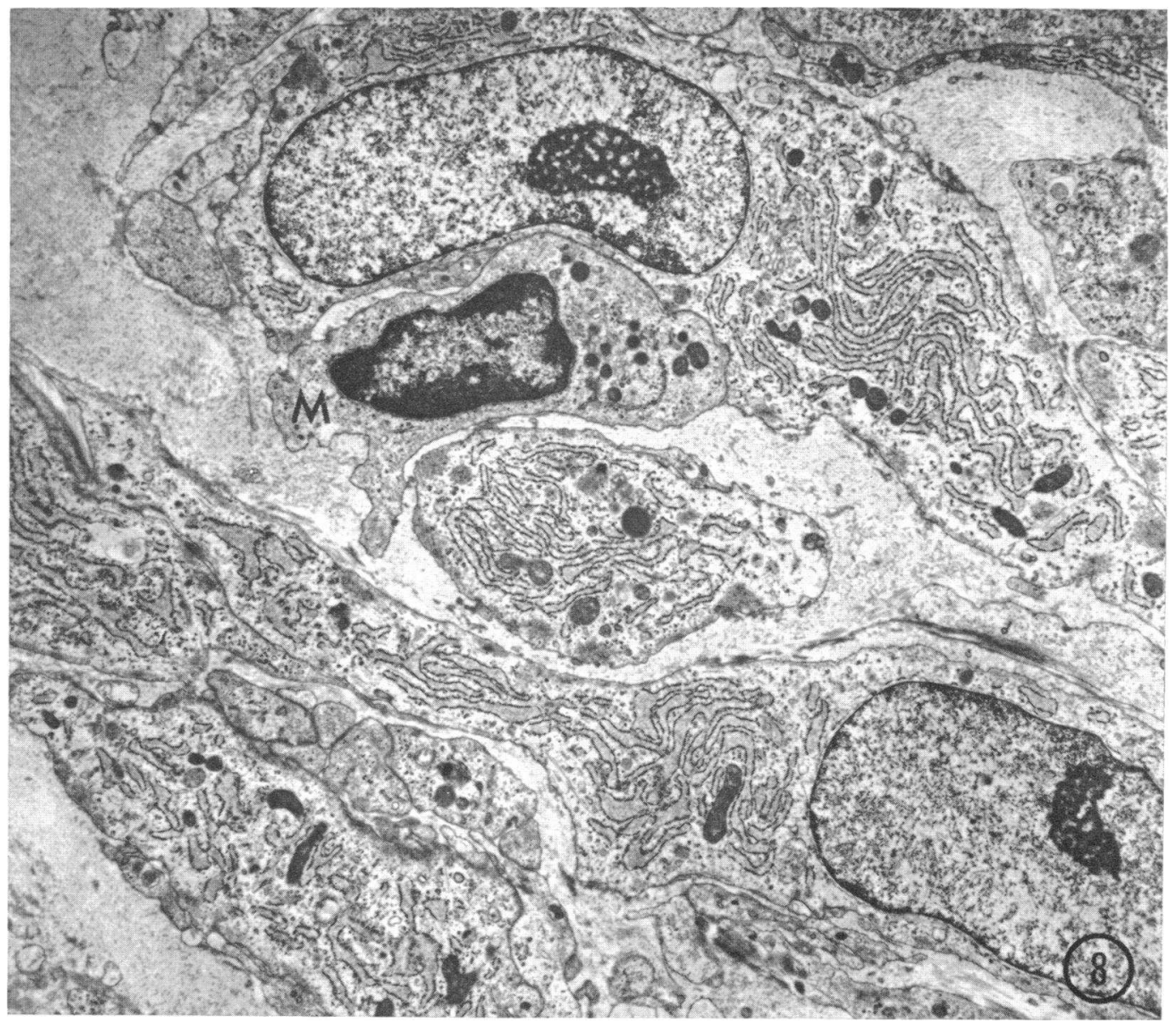

FIGURE 8 This electron micrograph is from an area within the center of a 10 day wound from a neutropenic guinea pig. At this time, both control and neutropenic wounds were characterized by the presence of numerous active fibroblasts and endothelial cells, as well as many new collagen fibrils within the intercellular spaces. The well-developed fibroblasts contain oval nuclei with prominent nucleoli, an extensive array of rough ER cisternae throughout the cytoplasm, and aggregates of small filaments just beneath the plasma membranes. A mononuclear phagocyte $(\mathrm{M})$ is also present. Magnification $\times 5700$.

from neutrophils participate in heterolysis, or clearance of tissue debris from the wound.

Recent investigators have continued to stress a causal relationship between inflammation and repair. Menkin (9) claimed that repeated injection of rabbits' ears with inflammatory exudates from the pleural cavity resulted in neoplastic growths. He suggested that this was a result of the presence of growth-promoting substances in leukocytes. The experiments of Selye (10) indicated that the nature and severity of inflammatory stimuli determine the extent of fibrosis in certain types of experimental injuries. Selye, Cunningham, Somogyi, and Cote (11) reported that agents such as croton oil, which cause the appearance of large numbers of neutrophils, seem to evoke an extensive formation of connective tissue and increased wound contraction.
A recent investigation by Alexander, Bossert, McClellan, and Altemeier (12) presented evidence for a role of substances released from lysosomes of neutrophils and other cell types in promoting wound repair. Yaron and Castor $(22,23)$ reported that human synovial fibroblasts in vitro could be "activated" by the addition of leukocytes to the media. Addition of either syngenic or allogenic lymphocytes or neutrophils led to increased glucose consumption, production of lactic acid, increased hyaluronic acid synthesis, and changes in fibroblast growth patterns. These results led them to speculate that infiltrating leukocytes release substances that stimulate fibroblasts in proliferative types of inflammatory responses, such as in rheumatoid arthritis.

It has not been known whether lysosomal enzymes or possibly other substances released by neutrophils are 
functional in debridement of the extracellular environment in wound exudates. However, it previously has been demonstrated that large numbers of neutrophils degenerate within wounds, releasing their cytoplasmic granules into the exudate $(1,21)$. In addition, the pathogenesis of tissue destruction in arthritic joints and in certain localized hypersensitivity reactions, such as the Arthus phenomenon, has been attributed to accumulation of neutrophils and release of enzymes or acid $(24,25)$.

The purposes of this study were: $(a)$ to examine whether the neutrophil, by its presence in the wound, provides any sort of initiating or stimulatory factor(s), humoral or otherwise, which promote influx of subsequent inflammatory cells or the repair process, resulting in scar formation. (b) To determine the importance of the neutrophil in debridement of the exudate in wounds, whether by phagocytosis, by extracellular release of hydrolytic enzymes, or both.

The most direct approach to these questions was provided by an examination of healing wounds devoid of neutrophils. Since neutrophils enter inflammatory sites from the local microcirculation, creation of a circulating neutropenia before wounding provided a means of preventing these cells from entering the wound. Most pharmacologic agents which deplete circulating leukocytes, such as nitrogen mustard or methotrexate (amethopterin), are toxic to most cells, especially rapidly proliferating populations, and would not be useful in such a study $(26,27)$. On the other hand, the use of antineutrophil serum provided a specific method for depleting circulating neutrophils with as little systemic trauma as possible (15). The animals tolerated the daily injections of ANS surprisingly well, and infection was not a major problem.

\section{Comparison of components in control and neutropenic wounds}

Acute inflammatory phase. Although the progress of wound repair in the neutropenic and control animals was histologically identical by the 7 th and 10th days, there were significant differences in the cellular components of these two groups of wounds at $24 \mathrm{hr}$. There were striking differences not only in the neutrophil content but in the volume of the wound occupied by red cells and space (presumably representing fluid) as well (Fig. 5). These differences were quantitated using $1-\mu$ Epon sections stained with basic fuchsin and methylene blue (18), which allowed the best possible recognition of components for histometric analysis. The recognition of cell types and other components was optimal under these conditions and in most sections there were less than $1 \%$ unidentifiable cells.

There was a much greater amount of "space" in the wounds of the controls as compared with the neutropenic animals. This difference appeared to have been accounted for by the greatly increased volume of red cells in the neutropenic wounds. One possible explanation for this is that substances released by neutrophils may assist in clearance of red cells by causing hemolysis. Hemolysis of the erythrocytes would release fluid and protein that would be counted as "space" using this type of analysis. Although some erythrocytes are removed by phagocytosis a larger number are probably cleared by hemolysis since the extent of erythrophagocytosis was not of sufficient magnitude to account for the total disappearance of red cells. Alternatively, the increased number of red cells in the neutropenic wounds could be the result of increased hemorrhage. It is also possible that a decreased fluid exudate in the neutropenic wounds may have accounted for part of the decrease in space that was observed. Factors that might result in a diminished exudate in neutropenic wounds could include the absence of vascular permeability-enhancing substances derived from neutrophils $(28,29)$ or a nonspecific anti-inflammatory effect resulting from transient depletion of complement levels subsequent to ANS, as suggested by previous investigators $(30,31)$.

Several enzymes that affect vascular permeability have been identified in neutrophil granules. These include a collagenase (32), an elastase (33), and a protease (34) all active at neutral $\mathrm{pH}$. In addition, a basic protein fraction derived from rabbit neutrophils has been shown to increase vascular permeability by two different mechanisms, one of which may be related to the release of histamine $(28,29)$.

Monocyte response. Significantly, there was no difference in the wound volume occupied by monocytes in 24-hr neutropenic and control wounds, nor were there any differences in the morphological appearance and phagocytic activity of the monocytes. Thus, emigration of neutrophils into wounds is clearly not an essential prelude to the emigration of mononuclear phagocytes. This finding is in contrast to the observations of Page and Good (35) who suggested that the emigration of neutrophils is essential to the subsequent appearance of mononuclear cells. They reported that inflammatory reactions elicited during the neutropenic phase of cyclic neutropenia or nitrogen mustard-induced neutropenia failed to develop the usual monocyte infiltration observed when circulating neutrophils were at normal levels. Recently, however, a similar study by Dale and Wolff (36) in patients with cyclic or chronic neutropenia indicated that the monocyte response in inflammation is not dependent upon a prior emigration of neutrophils. In an in vitro study utilizing the Boyden chamber, Ward (37) found that lysates of neutrophils are chemotactic for monocytes and suggested that this might provide the basis for monocyte emigration in vivo. Although neutrophils may 
contain factors chemotactic for monocytes in vitro, the observations in this study indicate that they are not essential for the emigration of monocytes into an inflammatory site such as a wound.

Connective tissue formation. The results of this study clearly demonstrate that, in the healing wound, the presence of neutrophils is not an essential antecedent to the invasion of fibroblasts and capillaries resulting in the orderly development of granulation tissue, and the subsequent formation of connective tissue. There were no observable differences in the extent of healing by 7-10 days in wounds from control and neutropenic animals. Although slight undetectable differences may exist between the extent of repair in the wounds from the two groups of animals, it is apparent that the healing process is in no way contingent upon a prior influx of neutrophils. The results presented here are in general agreement with those of several other investigations concerning the effects of experimental neutropenia on wound healing. In 1939, Lawrence, Pearse, and Mider (38) examined healing wounds in guinea pigs treated for up to 5 days with ANS. Although they observed a decreased mean tensile strength in neutropenic wounds compared with controls, they concluded that healing was not affected by the absence of neutrophils on the basis of their histologic observations. In 1966, Stein and Levenson (39) reported that the tensile strength and hydroxyproline content of 5-day wounds in rats was not affected by ANS administration. However, in both of these studies the wounds were observed for only 5 days and the degree of neutropenia was not clearly presented in the data. Wounds which have healed for only 5 days have just entered the fibroplastic phase (40), and the validity of tensile strength as a measure of the extent of repair at this time is questionable (41).

In a recent study of experimental myocardial infarcts in rats, Hill and Ward (42) found that in animals depleted of the third component of complement, the usual accumulation of neutrophils did not occur. This was apparently due to the absence of a chemotactic factor for neutrophils which is cleaved from C3 by a local tissue protease. These authors observed that in the absence of neutrophils there was no apparent reduction in the formation of granulation tissue during the healing of the infarct.

The animals that received ANS in this investigation were maintained in a neutropenic state throughout the 10 day course of wound repair. With few exceptions, all of the animals used in the study appeared healthy despite the low levels of circulating neutrophils. To keep the animals sufficiently neutropenic, it was necessary to give slightly increased daily doses of ANS after the 3rd day. This may have been necessitated by a combination of increased clearance of the antibody from the circulation, the mounting of an immune response against the antiserum or progressively increasing neutrophil production in the marrow.

Examination of bone marrow smears from ANS and NRS-treated animals at the termination of the 11 days of serum administration revealed an extreme proliferation of basophils and eosinophils in different stages of maturation. Other investigators have also reported that injections of foreign serum in guinea pigs results in increased marrow production and release of basophils $(43,44)$. In the present investigation it was found that the appearance of basophils in the wounds correlated with the onset of blood basophilia and eosinophilia, demonstrating that there was little or no cross-reactivity of ANS against these two types of granulocytes.

\section{Role of the neutrophils in wound healing}

Since the absence of neutrophils does not affect the progress of wound repair, it is reasonable to question the significance of the local accumulation of large numbers of these cells during the early stages of the healing process. The results of this study lend no credence to notions of earlier investigators of the importance of stimulatory substances or wound hormones in neutrophils. In addition, neutrophil enzymes released extracellularly are not essential in the clearance of wound debris. However, based on the increased volume of red cells in 24-hr neutropenic wounds as compared with controls, they may be important in the removal of red cells, possibly by hemolysis.

Neutrophils in uninfected wounds do not contribute significantly to wound clearance by phagocytosis. Some of the intact neutrophils observed in the electron microscope had small vacuoles containing fibrin or debris. Many of the neutrophils that entered the wounds underwent a series of degenerative changes that resulted in lysis and release of granules into the exudate. Most of the cells were ingested and removed by macrophages.

Clearly, the neutrophils in wounds and other sites of injury provide a principal agency for host defense against microorganisms. This crucial function has been known since the classical studies of Metchnikoff (45). Neutrophils are elegantly equipped to cope with ingested bacteria. Their cytoplasmic granules contain a potent armamentarium of hydrolytic enzymes and antibacterial substances $(46,47)$. The early arrival of large numbers of neutrophils in wounds thus provides an effective local barrier against bacterial invasion. No other role for this prominent early component of the inflammatory response was found in the healing wound. 


\section{ACKNOWLEDGMENTS}

The authors gratefully acknowledge the skilled technical assistance of Mrs. Claire Haney and the personnel of the Electron Microscope Laboratory.

This work was supported in part by U. S. Public Health Service Grants AM13970 and GM 00100.

\section{REFERENCES}

1. Ross, R., and E. P. Benditt. 1961. Wound healing and collagen formation. I. Sequential changes in components of guinea pig skin wounds observed in the electron microscope. J. Biophys. Biochem. Cytol. 11: 677.

2. Ross, R. 1968. The fibroblast and wound repair. Biol. Rev. (Camb.). 43: 51 .

3. Gillman, T. 1968. On some aspects of collagen formation in localized repair and in diffuse fibrotic reactions to injury. In Treatise on Collagen. B. S. Gould, editor. Academic Press, Inc., New York. 2 (Pt. B) : 331.

4. Arey, L. B. 1936. Wound healing. Physiol. Rev. 16: 327.

5. Edwards, L. C., and J. E. Dunphy. 1958. Wound healing. I. Injury and normal repair. N. Engl. J. Med. 259: 224

6. Carrel, A. 1921. Cicatrization of wounds. XII. Factors initiating regeneration. J. Exp. Med. 34: 425.

7. Carrel, A. 1924. Leukocytic trephones. J. Am. Med. Assoc. 82 : 255.

8. Carrel, A. 1922. Growth-promoting function of leucocytes. J. Exp. Med. 36: 385.

9. Menkin, V. 1941. Cellular injury in relation to proliferative and neoplastic responses. Cancer Res. 1: 548.

10. Selye, H. 1953. Part of inflammation in local adaptation syndrome. In International Symposium on the Mechanism of Inflammation. G. Jasmin and A. Robert, editors. Acta, Inc., Montreal. 53.

11. Selye, H., J. Cunningham, A. Somogyi, and G. Cote. 1969. Acceleration and inhibition of wound healing by topical treatment with different types of inflammatory irritants. Am. J. Surg. 117: 610 .

12. Alexander, J. W., J. E. Bossert, M. A. McClellan, and W. A. Altemeier. 1971. Stimulants of cellular proliferation in wounds. Arch. Surg. 103: 167.

13. Humphrey, J. H. 1955. The mechanism of Arthus reactions. II. The role of polymorphonuclear leucocytes and platelets in reversed passive reactions in the guinea pig. Br. J. Exp. Pathol. 36: 283.

14. Lawrence, J. S., C. G. Craddock, Jr., and T. N. Campbell. 1967. Antineutrophilic serum, its use in studies of white blood cell dynamics. J. Lab. Clin. Med. 69: 88.

15. Simpson, D. M., and R. Ross. 1971. Effects of heterologous antineutrophil serum in guinea pigs. Hematologic and ultrastructural observations. Am. J. Pathol. $65: 249$.

16. Miller, G. L., and J. E. Wilson. 1968. A study of variables in the assay of cytotoxic antisera. J. Immunol. $101: 1068$.

17. Karnovsky, M. J. 1965. A formaldehyde-glutaraldehyde fixative of high osmolality for use in electron microscopy. J. Cell Biol. 27: 137A.

18. Huber, J., F. Parker, and G. F. Odland. 1968. A basic fuchsin and alkalized methylene blue rapid stain for epoxy-embedded tissue. Stain Technol. 43: 83.

19. Weibel, E. R., G. S. Kistler, and W. F. Scherle. 1966. Practical stereological methods for morphometric cytology. J. Cell Biol. $30: 23$.
20. Scott, R. E., and R. G. Horn. 1970. Ultrastructural aspects of neutrophil granulocyte development in humans. Lab. Invest. 23: 202.

21. Ross, R., and G. Odland. 1968. Human wound repair. II. Inflammatory cells, epithelial-mesenchymal interralations, and fibrogenesis. J. Cell Biol. 39: 152.

22. Yaron, M., and C. W. Castor. 1969. Leukocyte-connective tissue cell interaction. I. Stimulation of hyaluronate synthesis by live and dead leukocytes. Arthritis Rheum. $12: 365$.

23. Castor, C. W., and M. Yaron. 1969. Leukocyte-connective tissue cell interaction. II. The specificity, duration, and mechanism of interaction effects. Arthritis Rheum. 12: 374 .

24. Weissman, G., I. Spilberg, and K. Krakauer. 1969. Arthritis induced in rabbits by lysates of granulocyte lysosomes. Arthritis Rheum. 12: 103.

25. Henson, P. M., and C. G. Cochrane. 1970. Cellular mediators of immunological tissue injury. J. Reticuloendothel. Soc. 8 : 124.

26. Staley, C. J., J. C. Kukral, and F. W. Preston. 1962. Effect of anticancer drugs on experimental wound healing. Surg. Forum. 13: 35.

27. Calnan, J., and A. Davies. 1965. The effect of methotrexate (amethopterin) in wound healing: an experimental study. Br. J. Cancer. 19: 505.

28. Janoff, A., S. Schaefer, J. Scherer, and M. A. Bean. 1965. Mediators of inflammation in leukocyte lysosomes. II. Mechanism of action of lysosomal cationic protein upon vascular permeability in the rat. J. Exp. Med. 122: 841 .

29. Ranadive, N. S., and C. G. Cochrane. 1968. Isolation and characterization of permeability factors from rabbit neurophils. J. Exp. Med. 128: 605.

30. Willoughby, D. A., E. Coote, and J. L. Turk. 1969. Complement in acute inflammation. J. Pathol. $97: 295$.

31. Willoughby, D., and W. Spector. 1968. Inflammation in agranulocytotic rats. Nature (Lond.). 219: 1258.

32. Lazarus, G. S., R. S. Brown, J. R. Daniels, and H. M. Fullmer. 1968. Human granulocyte collagenase. Science (Wash., D. C.). $159: 1483$.

33. Janoff, A. 1970. Mediators of tissue damage in leukocyte lysosomes. Further studies on human granulocyte elastase. Lab. Invest. 22 : 228.

34. Janoff, A., and J. D. Zeligs. 1968. Vascular injury and lysis of basement membranes in vitro by neutral protease of human leukocytes. Science (Wash., D. C.). $161: 702$.

35. Page, A. R., and R. A. Good. 1958. A clinical and experimental study of the function of neutrophils in the inflammatory response. Am. J. Pathol. 34: 645.

36. Dale, D. C., and S. M. Wolff. 1971. Skin window studies of the acute inflammatory responses of neutropenic patients. Blood J. Hematol. 38: 138.

37. Ward, P. A. 1968. The chemotaxis of mononuclear cells. J. Exp. Med. 128 : 1201.

38. Lawrence, J. S., H. E. Pearse, and G. B. Mider. 1939. Effect of experimental neutropenia on the healing of wounds. Arch. Pathol. 28: 32.

39. Stein, J. M., and S. M. Levenson. 1966. Effect of the inflammatory reaction on subsequent wound healing. Surg. Forum. 17 : 484.

40. Dunphy, J. E., and K. N. Udupa. 1955. Chemical and histochemical sequences in the normal healing of wounds. N. Engl. J. Med. 253 : 847. 
41. Van Winkle, W., Jr. 1969. The tensile strength of wounds and factors that influence it. Surg. Gynecol. Obst. 129 : 819.

42. Hill, J. H., and P. A. Ward. 1971. The phlogistic role of C3 leukotactic fragments in myocardial infarcts of rats. J. Exp. Med. 133: 885 .

43. Chan, B. S. T., and J. M. Yoffey. 1960. The basophil cells of guinea pig bone marrow and their response to horse serum. Immunology. 3: 237.

44. Winqvist, G. 1960. Experimental production of basophil granulocytes in the guinea pig. Exp. Cell Res. 19: 7.
45. Metchnikoff, E. 1891. Lectures on the Comparative Pathology of Inflammation. Translated from the French by F. A. Starling and E. H. Starling, editors. Dover Publications, Inc., New York. 180.

46. Hirsch, J. G. 1965. Neutrophil and eosinophil leukocytes. In The Inflammatory Process. B. W. Zweifach, L. Grant, and R. T. McCluskey, editors. Academic Press, Inc., New York. 245.

47. Cohn, Z. A., and J. G. Hirsch. 1960. The isolation and properties of the specific cytoplasmic granules of rabbit polymorphonuclear leukocytes. J. Exp. Med. 112: 893. 